\title{
Metastatic breast cancer: an unusual cause of diplopia
}

\author{
Nasser Mohammed Amer ${ }^{1}$, Gareth Bashir ${ }^{2}$, Arikoge Ogedegbe ${ }^{3}$, Ibtisam Saeed ${ }^{4}$ \\ ${ }^{I}$ Department of General Surgery, University of Dammam, Al Khobar 40262, Kingdom of Saudi Arabia. \\ ${ }^{2}$ Department of General Surgery, King George Hospital, Ilford IG3 8YB, UK. \\ ${ }^{3}$ Department of General Surgery, King George Hospital, Ilford IG3 8YB, UK. \\ ${ }^{4}$ Department of Pathology, Queens Hospial, Romford RM7 OAG, UK.
}

Correspondence to: Dr. Nasser Mohammed Amer, Department of General Surgery, University of Dammam, A1 Khobar 40262, Kingdom of Saudi

Arabia. E-mail: nmamer@uod.edu.sa

\section{A B S T R A C T}

While secondary solid cancer into the eye orbit is rare, it is the most common site for primary metastasis in female breast cancer. We report a case of a sixty-six years old woman presenting to her optician with complaints of double vision. Magnetic resonance imaging revealed an invasive lesion in the superior and medial rectus muscles of the right orbit, biopsy of which confirmed this as an infiltrating breast carcinoma. Investigation of the primary lesion showed an advanced invasive ductal carcinoma of the right breast. She was then treated with radiotherapy to the orbit and a non steroidal aromatase inhibitor Anastrozol (Arimidex ${ }^{\circledR}$ ). We herein review and discuss the literature, epidemiology, mechanism of tumor spread, the "seed and soil" theory, clinical presentation, pathology, and management of this uncommon presentation.

Key words: Metastatic breast cancer; diplopia; ocular metastasis

\section{INTRODUCTION}

Cancer metastasis to the eye orbit is rare ${ }^{[1-4]}$ However, female breast cancer is the commonest primary cancer metastasizing to the orbit, ${ }^{[2,3,5,6]}$ followed by, lung cancer, prostate cancer, melanoma, and genitourinary cancer in no particular order. Patients typically present with limited ocular mobility, ${ }^{[6]}$ proptosis, blepharoptosis, palpable mass, blurred or decreased vision or pain. Signs and symptoms relating to orbital metastasis are usually noted late in the disease progression, and treatment generally consists of local radiotherapy to the orbit ${ }^{[6]}$ in addition to treatment of the primary cancer, which in this case of ductal adenocarcinoma of the breast required hormonal therapy only.

\section{CASE REPORT}

A 66-year-old woman presented to her optician with symptoms of diplopia affecting the right eye only and was subsequently referred to an ophthalmologist. Physical examination showed a right sided ptosis and significant impairment in all extra-ocular muscle function with some

\begin{tabular}{|l|l|}
\hline \multicolumn{3}{|c|}{ Access this article online } \\
\hline Quick Response Code: & Website: \\
& http://jcmtjournal.com \\
\cline { 2 - 2 } & \\
\hline
\end{tabular}

sparing of the lateral rectus and superior oblique muscles. The initial diagnosis was that of a partial third cranial nerve palsy. A computed tomography (CT) scan revealed increased abnormal soft tissue enhancement in the superior aspect of the orbit, with involvement of superior and medial rectus muscles. The patient was then referred to an oculoplastic surgeon who noted that the patient had a "frozen eyeball". A magnetic resonance imaging (MRI) scan further revealed an abnormal infiltrating lesion at the orbital apex encasing the optic nerve and involving all four rectus muscles [Figures 1 and 2]. Next, a CT-guided biopsy of the lesion was performed and histology revealed a metastatic lesion, most likely from a primary breast adenocarcinoma [Figure 3]. Thus, the patient was referred to our multidisciplinary breast clinic whereupon a $10 \mathrm{~mm}$ palpable mass in the upper outer quadrant of the right breast was found, clinically suspicious of cancer. A mammogram and ultrasound were performed, followed by a core biopsy of the mass. The latter demonstrated features consistent with an invasive ductal carcinoma, histologically identical to the biopsy from the orbit. The tumor cells were estrogen receptor

This is an open access article distributed under the terms of the Creative Commons Attribution-NonCommercial-ShareAlike 3.0 License, which allows others to remix, tweak, and build upon the work non-commercially, as long as the author is credited and the new creations are licensed under the identical terms.

For reprints contact: service@oaepublish.com

How to cite this article: Amer NM, Bashir G, Ogedegbe A, Saeed I. Metastatic breast cancer: an unusual cause of diplopia. J Cancer Metastasis Treat 2016;2:123-6.

Received: 10-02-2015; Accepted: 29-12-2015. 


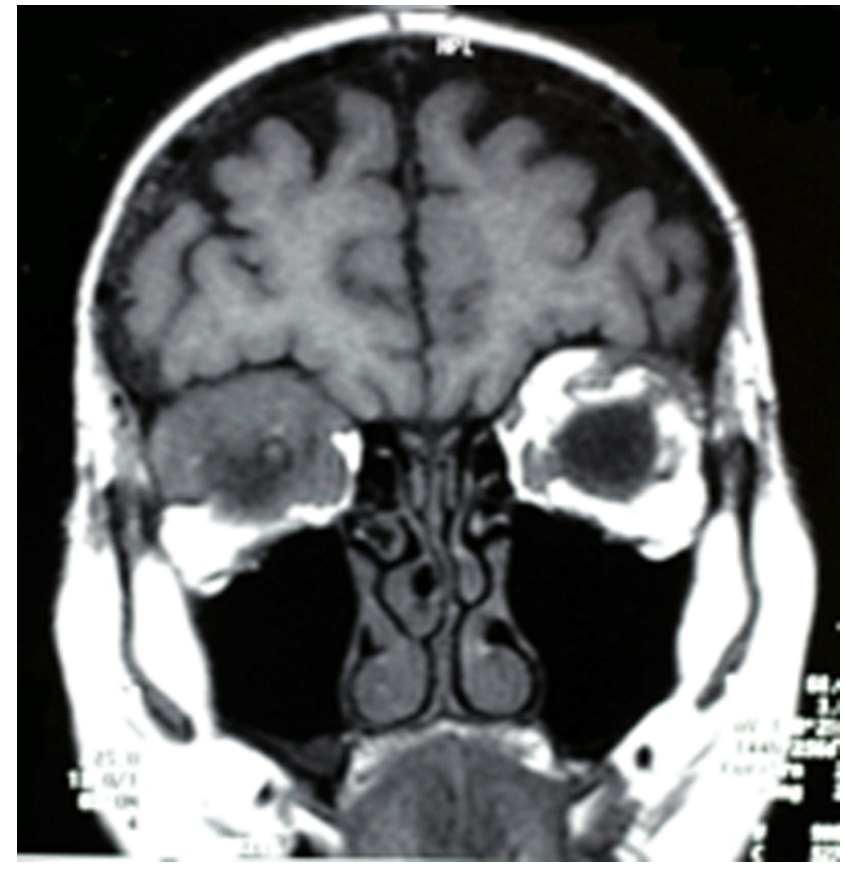

Figure 1: Axial T1 MRI of skull showing an abnormal infiltrating lesion at the right orbital apex involving all four rectus muscles

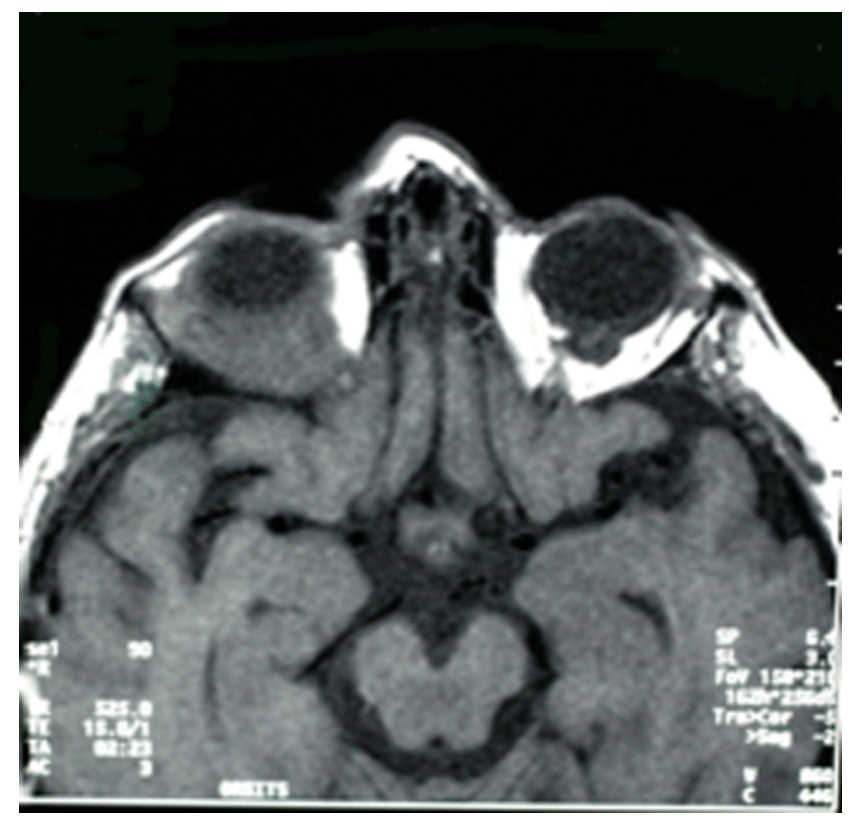

Figure 2: Coronal T1 MRI of skull shows intra- and extra-conal soft tissue signal intensity lesion encroaching upon right optic nerve, likely infiltrating and effacing retrobulbar fat

positive, progesterone receptor positive and HER2 negative (Luminal A). Staging investigation unfortunately revealed diffuse bony metastases. The consensus of our team was to treat her with primary hormonal therapy and the patient was treated with an aromatase inhibitor (Arimidex ${ }^{\circledR}$ ) $1 \mathrm{mg}$ once a day. Adjuvant radiotherapy to the right eye was also done under the direction of the ocular oncologist.

\section{DISCUSSION}

Orbital metastasis of solid tumors is very rare ${ }^{[2-6]}$ with most occurring in the uveal tract, especially in the posterior

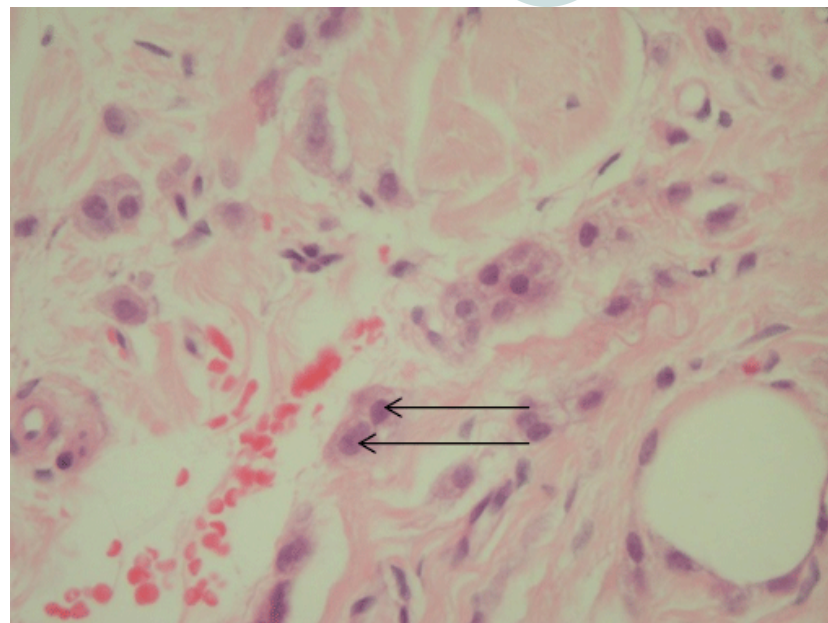

Figure 3: Histology of orbital core biopsy showing infiltration by metastatic adenocarcinoma, most likely of breast origin

part of the choroid. It has been proposed ${ }^{[7]}$ that breast cancer cells have the ability to remain viable away from their primary site. Indeed, breast cancer accounts for the highest incidence of metastasis to the orbit, ${ }^{[2,3,5-9]}$ followed by prostatic, lung, melanoma, and gastrointestinal cancers. Another study showed that lung cancer was the second most common primary source for orbital metastasis, followed by prostate cancer. Other reported sources include cancers of the thyroid, liver, pancreas, adrenal gland, salivary and choroidal melanoma. ${ }^{[6]}$

Overall, orbital metastasis occurs in $2-3 \%$ of patients with systemic cancer ${ }^{[6]}$ However, metastasis into the extraocular muscles is an even less frequent presentation. ${ }^{[1]}$ It did, however, occur in this reported case of breast carcinoma metastatic to the orbit, with all four recti muscles being involved. This is not in concordance with the prevailing view that skeletal muscles are considered an uncommon site for metastasis, (albeit less infrequent in malignant lymphoma and leukemia). It may be due to the fact that these muscles are in a more or less constant state of movement, thus preventing neoplastic cells from seeding them, or by producing an unfavorable chemical environment for neoplastic growth. ${ }^{[5]}$ Clinical studies have shown that different cancer types frequently display distinct metastatic patterns, with neoplasms of particular histological types tending to metastasize to specific organs. ${ }^{[10]}$ Paget $^{[11]}$ first proposed the "seed and soil" hypothesis of cancer metastasis. He postulated that tumor development was a consequence of the provision of a fertile environment (the soil) in which compatible tumor cells (the seed) could proliferate The ability or inability of specific organs to provide this favorable milieu and the success or failure of specific cells to respond to these microenvironments dictated the observed patterns of metastatic development in different cancers. ${ }^{[11]}$ In contrast to the "seed and soil" theory, there is a mechanistic explanation for secondary tumor growth patterns. Here, the organ or tissue specificity is the direct consequence of the anatomical location of primary tumors. Thus, the secondary foci of epithelial cancers, 
which metastasize predominantly via the lymphatics, are subsequently found mainly in draining lymph nodes. It has been proposed that entrapment and growth of tumors might be affected by qualitative or quantitative differences in tumor cells' ability to adhere to the vascular endothelium of particular organs; ${ }^{[11]}$ organ-specific growth is thus a direct consequence of the specific localization or entrapment of circulating tumor cells. While it is tempting to speculate an immunologic basis for the propensity of breast cancer and malignant melanoma to metastasize to the extra-orbital muscles; the nature of any such site specificity remains unknown. ${ }^{[2]}$

Although the patient did have skeletal symptoms due to widespread bony metastasis which were largely dismissed by her general practitioner as being part of her arthritis complex, the main presenting symptom in this case was diplopia. However, a review of the literature indicates that this not commonly the case. A previous study of 100 patients $^{[6]}$ showed that diplopia constituted only $9 \%$ of such patients. Frequency of their presenting symptoms and signs were limited ocular mobility (54\%), displacement of the globe with proptosis $(50 \%)$, blepharoptosis $(49 \%)$, a palpable mass (43\%), blurred or decreased vision $(23 \%)$, pain $(17 \%)$, visible mass or swelling $(17 \%)$, and enophthalmos $(11 \%)$. The latter sign interestingly and paradoxically was found to be associated with scirrhous breast cancer. ${ }^{[6,9,12]}$ This may be explained by the presence of desmoplasia and fibrosis associated with the tumor, causing contracture of the orbital content, and paradoxical enophthalmous.

The diagnosis of metastatic tumor can be confirmed by CT $\operatorname{scan}^{[1,2]}$ for more than $95 \%$ of orbital metastases and CT scan can provide considerable information regarding size, location, relation to musculature and other structures, as a well as the nature of the lesion. High resolution CT imaging is also an excellent diagnostic tool for extra-ocular metastasis. Focal or nodular muscle enlargement without focal bone destruction, fossa formation, orbital enlargement, or other evidence of neoplastic extension into contiguous structures is highly suggestive of metastasis. Diffuse enlargement with feathering of the muscle edge may occur or masquerade clinically as a myositic pseudotumor. ${ }^{[6]}$ Bilateral involvement can be present despite only unilateral symptoms. ${ }^{[6]} \mathrm{MRI}$, on the other hand, did not add specificity to the radiographic information in our case. Any patient with an undifferentiated malignancy first discovered in the orbit should undergo a full systemic evaluation to reveal the primary tumor. Although MRI may provide the best resolution of orbital metastasis, ${ }^{[6]} \mathrm{CT}$ is more useful in cases of suspected prostate and breast cancer as metastatic bone involvement is very common in breast cancer too.

Metastatic lesions involved the horizontal rectus muscles are more common than the vertical rectus or oblique muscles. ${ }^{[6]}$ Orbital metastasis from breast cancer tends to be diffuse and irregular, often growing along rectus muscles and fascial planes. In contrast, orbital metastasis from carcinoid tumor, renal cell carcinoma, and melanoma tends to be more circumscribed, at least in early stages. ${ }^{[6]}$ Diagnosis is usually established by tissue biopsy, open, or fine needle aspiration to confirm a metastatic tumor lesion, and not simulating lesions, such as idiopathic orbital inflammation (also known as orbital pseudotumor). Special staining ${ }^{[12]}$ with mucicarmine and alcian blue may be helpful, not only to obtain a diagnosis but also to identify the primary neoplasm. Histological diagnosis of metastasis is typically straightforward and does not present a problem to the pathologist. ${ }^{[7]}$

Although we did not test for Ki-67 status, (also known as MK 167) it has been used as prognostic parameter in breast cancer patients, ${ }^{[14]}$ and its presence is associated with lower disease free survival and lower overall survival. Currently neither St Gallen, nor ASCO recommendations nor the German Interdisciplinary S3 Guidelines for the diagnosis, treatment, and follow-up of breast cancer have proposed Ki-67 as a routine prognostic marker. Ki-67 has been shown to have an inverse relationship with estrogen receptor status, but a possible direct relationship with HER2 status.

Treatment of histologically proven metastatic tumors to the orbit is mainly palliative, ${ }^{[12]}$ and is comprised of orbital irradiation ${ }^{[6]}$ with approximately $35-40$ Gy to the affected orbit in divided doses over 3-5 weeks. Slightly more or less radiation may be indicated depending on tumor type. Radiation $^{[7]}$ may improve and preserve vision for the remaining lifespan of the patient. Regression of ocular metastasis following sterilization, adrenalectomy, or hypophysectomy has been reported in a number of cases of hormone dependent breast cancers, ${ }^{[2]}$ however, Aromatase inhibitors (Anastrozole, Letrozole, Exemestane) are now recognized as the agents of choice for the management of post menopausal women with steroid hormone positive metastatic breast cancer, in whom indications for chemotherapy are not absolute. ${ }^{[13]}$ Enucleation of the eye on the other hand should only be carried out in cases of intractable pain, most often caused by secondary glaucoma. ${ }^{[2]}$

\section{Acknowledgments}

The author would like to thank Dr Mohammed Azazi, Consultant Radiologist, King Fahad University Hospital, Al Khobar, KSA, for his assistance in interpreting the images, and Dr I. T Saed, FRC path, Consultant Histopathologist, Queens Hospital, Romford UK, for her contribution to the slides.

\section{Financial support and sponsorship}

Nil.

\section{Conflicts of interest}

There are no conflicts of interest. 


\section{REFERENCES}

1. Weiss R, Grisold W, Jellinger K, Mühlbauer J, Scheiner W, Vesely M. Metastasis of solid tumors in extraocular muscles. Acta Neuropathol (Berl) 1984;65:168-71.

2. Capone A Jr, Slamovits TL. Discrete metastasis of solid tumors to extraocular muscles. Arch Ophtalmol 1990;108:237-43

3. van der Heijden A, Twijnstra A, Lamers WP, Hupperets PS, Freling G. An unusual case of diplopia in a cancer patient. Eur J Cancer 1991;27:1315-6.

4. Cuttone JM, Litvin J, McDonald JE. Carcinoma metastatic to an extraocular muscle. Ann Ophthal 1981;13:213-6.

5. Ashton N, Morgan G. Discrete carcinomatous metastases in the extraocular muscles. Br J Ophthalmol 1974;58:112-7.

6. Shields JA, Shields CL, Brotman HK, Carvalho C, Perez N, Eagle RC Jr. Cancer metastatic to the orbit, the 2000 Robert M. Curts Lecture. Ophthal Plast Reconstr Surg 2001;17:346-54.

7. Jensen OA. Metastatic tumours of the eye and orbit. A histopathological analysis of a Danish series. Acta Pathol Microbiol Scand Suppl 1970;212:Suppl 212:201+.
8. Shields JA, Shields CL, Scartozzi R. Survey of 1264 patients with orbital tumors and simulating lesions. The 2002 Montgomery Lecture, part 1. Ophthalmology 2004;111:997-1008.

9. Mortada A. Binocular diplopia due to metastasis in orbital muscle from female breast cancer years after radical mastectomy. Orbit 1984;3:71-3.

10. Hart IR. 'Seed and soil' revisited: mechanisms of site-specific metastasis. Cancer Metastasis Rev 1982;1:5-16.

11. Paget S. The distribution of secondary growths in cancer of the breast. 1889. Cancer Metastasis Rev 1989;8:98-101.

12. Font RL, Ferry AP. Carcinoma metastatic to the eye and orbit III. A clinicopathologic study of 28 cases metastatic to the orbit. Cancer 1976;38:1326-35.

13. Wickremasinghe S, Dansingani KK, Tranos P, Liyanage S, Jones A, Davey C. Ocular presentation of breast cancer. Acta Ophthalmol Scand 2007;85:133-42.

14. Inwald EC, Klinkhammer-Schalke M, Hofstädter F, Zeman F, Koller M, Gerstenhauer M, Ortmann O. Ki-67 is a prognostic parameter in breast cancer patients: result of large population base cohort of a cancer registry. Breast Cancer Res Treat 2013;139:539-52. 